\title{
Fuzzy Optimisation of Structural Performance
}

M. Holický

Structural performance has become a fundamental concept in advanced engineering design in construction. Basic criteria concerning the action effects and imposed performance requirements are analysed assuming two types of uncertainties: randomness and vagueness. The natural randomness of the action effect is handled by commonly used methods of the theory of probability. The vagueness of performance requirements due to indistinct or imprecise specification and perception is analysed using the basic tools of the theory of fuzzy sets. Both types of uncertainties are considered to define fuzzy probabilistic measures of structural performance, the damage function and fuzzy probability of failure. Fuzzy probabilistic optimisation of vibration constraints indicates that the limiting values recommended for the acceleration of building structures may be uneconomical. Further research should focus on verifying of the input theoretical models using available experimental data.

Keywords: performance requirements, randomness, fuzziness, optimisation.

\section{Notation}

C Total cost

$C_{0} \quad$ Construction and maintenance cost

$C_{\mathrm{D}} \quad$ Cost of full damage (full malfunction or performance failure)

$D_{R}(x)$ Damage function

$F(z, t)$ Load function of a generic point $z$ and time $t$

$K(z) \quad$ Deflection of a beam

$k \quad$ Constant

$N \quad$ Normalising factor

$S \quad$ Action effect

$R \quad$ Resistance

$r_{1} \quad$ Lower bound of the transition region

$r_{2} \quad$ Upper bound of the transition region

$x \quad$ A generic point of a relevant performance indicator

$z \quad$ A point of a beam

$t \quad$ Time point

$\pi \quad$ Fuzzy probability of performance failure

$\xi \quad$ Decision parameter

$\mu_{R} \quad$ The mean of $\mathrm{R}$

$\sigma_{R} \quad$ The standard deviation of $\mathrm{R}$

$\mu_{S} \quad$ The mean of $\mathrm{S}$

$\sigma_{S} \quad$ The standard deviation of $\mathrm{S}$

$v_{R}(x) \quad$ Membership function

$\varphi_{R}(x \mid v)$ Probability density function

$v(z, t) \quad$ Deflection at a generic point $\mathrm{z}$ and time $\mathrm{t}$

EJ Stiffness of a beam

\section{Introduction}

Structural performance has become a fundamental concept in advanced engineering design in construction. However, the performance requirements (including serviceability, safety, security, comfort, functionality) of buildings and engineering works are often affected by various uncertainties that can hardly be entirely described by traditional probabilistic models. As a rule, the transformation of human needs and desires, particularly of those describing occupancy comfort and aesthetical aspects, to performance (user) requirements often results in an indistinct or imprecise specification of the technical criteria for relevant performance indicators (for example permissible deflection, crack width, velocity, acceleration) [1].

Thus, in addition to the natural randomness of basic variables, the performance requirements may be considerably affected by vagueness in the definition of technical criteria. Two types of uncertainty of performance requirements are therefore identified here: randomness, handled by commonly used methods of the theory of probability, and fuzziness, described by the basic tools of the recently developed theory of fuzzy sets [2, 3]. Similarly as in previous studies [4, 5], the fundamental condition of structural performance, $S \leq R$, relating an action effect $S$ and a relevant performance requirement $R$, is analysed assuming the randomness of $S$ and both the randomness and the fuzziness of $R$.

An illustrative example of continuous vibration in offices is used throughout the paper to clarify the general concepts. In this example, it is shown that it is impossible to identify a distinct value of an appropriate indicator (a root mean square value of acceleration) that would separate satisfactory performance from unsatisfactory performance (see also $[6,7,8]$ ). Typically, a broad transition region is observed, where the building gradually loses its ability to perform adequately and where the degree of damage (inadequate performance or malfunction) gradually increases. This paper is an extension of previous studies $[4,5,9,10]$.

\section{Theoretical model for performance requirements}

Fuzziness due to vagueness and imprecision in the definition of performance requirement $R$ is described by the membership function $v_{R}(x)$ indicating the degree of membership of a structure in a fuzzy set of damaged (unserviceable) structures $[1,4,5]$; here $x$ denotes a generic point of a relevant performance indicator (in the illustrative example the root mean square value of acceleration) used to assess both $S$ and $R$. A simple piecewise linear membership function $v_{R}(x)$, shown in Fig. 1, is considered in the following analysis. This function 


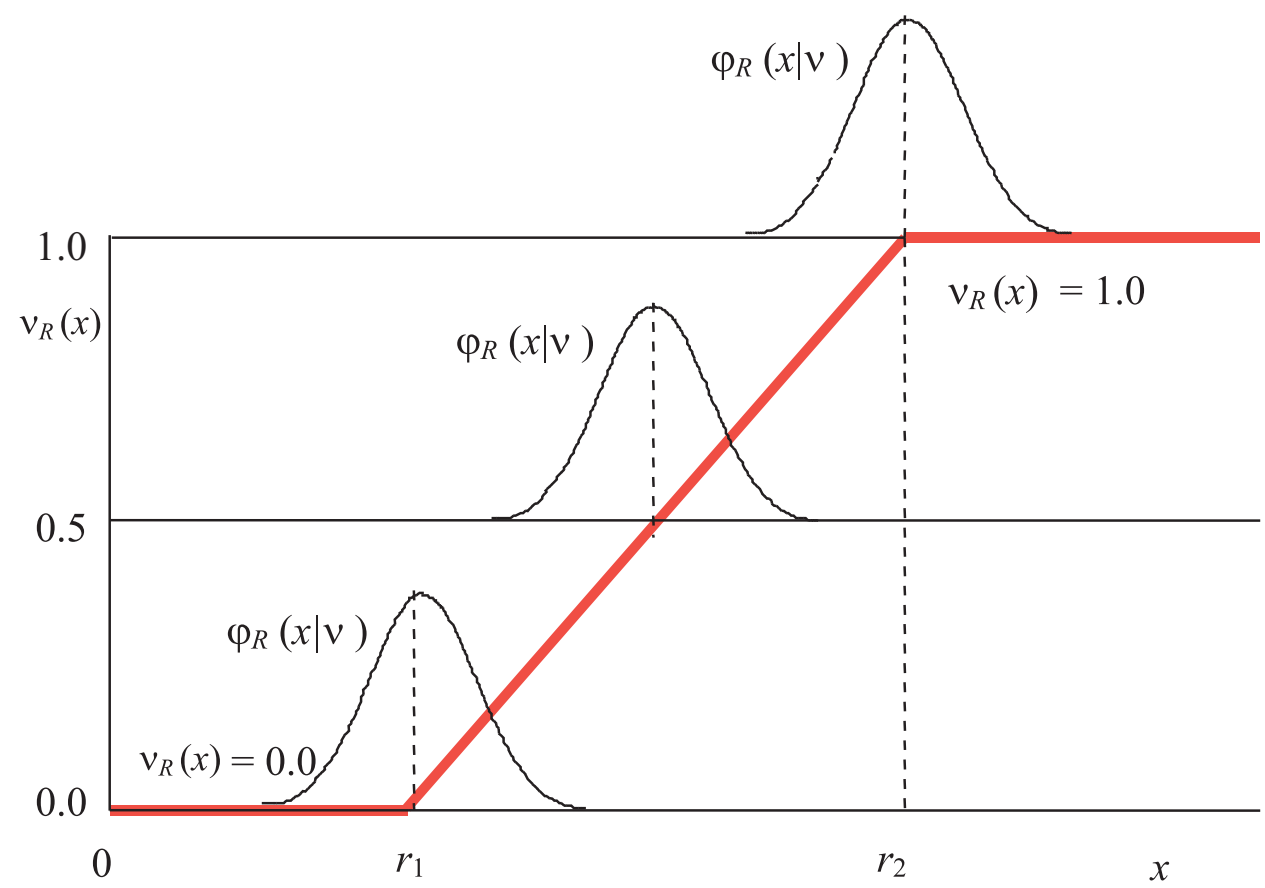

Fig. 1: Fuzzy probabilistic model of the performance requirement $R$

describes the non-random (deterministic) part of uncertainty in the requirement $R$. The randomness of $R$ at each damage level $v=v_{R}(x)$ is described by the probability density function $\varphi_{R}(x \mid v)$ (see Fig. 1), for which the normal distribution is considered here.

The transition region $\left\langle r_{1}, r_{2}\right\rangle$, where the building gradually loses its ability to perform adequately and its damage increases, may be rather broad depending on the nature of the performance requirement. In the illustrative example of continuous vibration in offices, the upper bound $r_{2}$ may be a multiple of the lower bound $r_{1}$ (see the International Standards [6, 7] and the comprehensive discussion in [8]). An assessment of the lower bound $r_{1}$ can be derived from the root mean square value of the acceleration limits, which are expected to be approximately equal to $r_{1}$.

The acceleration constraints for continuous vibration in offices suggested in various countries (see the critical review in [8]) are within a range from 0.02 to $0.06 \mathrm{~ms}^{-2}$. As discussed in [8], the vibration threshold may be even lower, within values from 0.01 to $0.02 \mathrm{~ms}^{-2}$. In the case of continuous vibration in offices there is, however, a low probability of an adverse comment for accelerations below $0.02 \mathrm{~ms}^{-2}$ [8]. Therefore, this value may be considered as the lower limit $r_{1}$ below which an office is assumed to be fully serviceable and to perform adequately.

The assessment of the upper limit $r_{2}$, above which an office is fully unserviceable, is even more difficult than the appraisal of the lower limit $r_{1}$. The upper limit $r_{2}$ may vary considerably depending on the definition of a fully unserviceable state of a building. In accordance with the discussion in [8], an adverse comment is probable for accelerations above $0.10 \mathrm{~ms}^{-2}$. Although this value may not imply a full disability of a building space to be used as an office, it is accepted here as an assessment of the upper limit $r_{2}$. To show the effect of the upper limit of the transition region on optimum constraints, two indicative values $r_{2}=3 r_{1}\left(=0.06 \mathrm{~ms}^{-2}\right)$ and $r_{2}=5 r_{1}\left(=0.10 \mathrm{~ms}^{-2}\right)$ are considered in the following analysis.
In addition to fuzziness, performance requirements are also dependent on the natural randomness of user needs. As already indicated above, this uncertainty is described in Fig. 1 by the normal probability density function $\varphi_{R}(x \mid v)$. The mean of $\varphi_{R}(x \mid v)$ for a given damage level $v$ is considered as the value of the indicator $x$ for which $v=v_{R}(x)$, the standard deviation is taken as independent of $x$ and equal to $0.1 r_{1}\left(0.002 \mathrm{~ms}^{-2}\right)$.

The above described theoretical model of performance requirements, including the fuzziness and randomness characteristics, should however be considered only as a conceivable representation of actual user needs. In order to determine a more accurate and more precise fuzzy probabilistic model of performance requirements, there is an urgent need for further development in the definitions of newly introduced characteristics of performance uncertainties using appropriate experimental data.

\section{Theoretical model of public perception}

The fuzzy probabilistic concepts introduced above may be effectively used as a theoretical model of public perception $[9,10]$. Public perception plays an important role in the assessment and final decision concerning any existing structure. Due to its performance deficiency the new department store described in [9] soon became a building closely watched by a large number of users and local authorities.

After a few years in service serious performance defects of the cladding, interior partitions, and other secondary elements were observed [9]. Incidentally, at the same time another department store suffered from construction faults and this was partly the reason why all the deficiencies have been carefully recorded. This unfavourable engineering climate seems to enhance the intensity of public perception. The observed defects were often exaggerated and regarded as indicators of insufficient structural safety. Widespread public perception of the defects and discrepancies in expert assess- 
ments was reported in the newspapers and finally resulted in a strong public demand for the building to be strengthened [9].

The evaluation of public as well as expert assessments confirms the fundamental fuzzy probabilistic concepts described above. There is no distinct point in any commonly used performance indicator $x$ (e.g. deflection, crack width) that would uniquely separate acceptable and unacceptable structures. Rather, there seems to be a transition region in which the structure gradually becomes unserviceable and the degree of caused damage $v_{R}(x)$ increases. It appears that the conceivable model for $v_{R}(x)$ indicated in Fig. 1 may be used for the description of public perception [10]. Note that the values of $v_{R}(x)$ are within the conventional interval from 0 to 1 . In the assessment of existing structures there is no damage below a certain lower limit value $r_{1}$, and full damage above the upper limit $r_{2}$.

The fuzzy probabilistic measures developed below can be used for the analysis of an structural performance of new structures as well as for an assessment of the public perception of existing structures exhibiting performance deficiencies.

\section{Fuzzy probabilistic measures of structural performance}

The damage function $D_{R}(x)$ is defined as the weighted average of damage probabilities reduced by the corresponding damage level $[4,5]$

$$
D_{R}(x)=\frac{1}{N} \int_{0}^{1} v\left(\int_{-\infty}^{x} \varphi_{R}\left(x^{\prime} \mid v\right) \mathrm{d} x^{\prime}\right) \mathrm{d} v,
$$

where $N$ denotes a factor normalising the damage function $D_{R}(x)$ to the conventional interval $\langle 0,1\rangle$ and $x^{\prime}$ is a generic point of $x$. The damage function $D_{R}(x)$ defined by equation (1) may be used $[4,5]$ to specify the design (or characteristic) value of the performance requirements corresponding to a given level of the total expected damage. Thus, for the fuzziness characteristics $r_{1}$ and $r_{2}$, and the randomness characteristic $\sigma_{R}$ the design value of the performance requirement $R$ may be specified in a rational way using fuzzy probabilistic concepts.

The fuzzy probability of performance (serviceability) failure $\pi$ is then defined as $[4,5]$

$$
\pi=\int_{-\infty}^{\infty} \varphi_{S}(x) D_{R}(x) \mathrm{d} x
$$

where $\varphi_{S}(x)$ is the probability density function of the action effect $S$. Similarly the fuzzy probability of performance failure $\pi$ defined by equation (2) enables the formulation of various design criteria in terms of relevant randomness as well as fuzziness characteristics. Then, however, besides the fuzziness characteristics $r_{1}, r_{2}$ and the randomness characteristic $\sigma_{R}$ of the performance requirement $R$, the characteristics of action effect $S$, particularly the mean $\mu_{S}$ and the standard deviation $\sigma_{S}$ are also needed. In the following, a symmetric normal (Laplace-Gauss) distribution of $S$ is accepted. The general case of an asymmetric three parameter lognormal distribution is considered in earlier studies $[4,5]$.

\section{Optimisation procedure}

The optimum value of the fuzzy probability of performance failure can be estimated using the technique of design optimisation $[4,5]$. It is assumed that the objective function is given by the total $\operatorname{cost} C(\xi)$ expressed approximately as a sum

$$
C(\xi)=C_{0}(\xi)+\pi(\xi) C_{D},
$$

where $C_{0}(\xi)$ is given as the sum of the construction and maintenance cost, $\pi(\xi) C_{\mathrm{D}}$ is the expected malfunction cost; here $C_{\mathrm{D}}$ denotes the cost of full damage (full malfunction or serviceability failure) and $\xi$ denotes the decision parameter (for example the mass per unit length or the cross section area). It has been shown $[4,5]$ that this equation can be used if the malfunction cost due to the damage level $v$ is given as the multiple $v C_{\mathrm{D}}$ (in the illustrative example this represents the cost due to disturbance and the lower efficiency of occupancies in the offices). Further, it is assumed that both the initial cost $C_{0}(\xi)$ and the fuzzy probability of performance failure $\pi(\xi)$ are dependent on a decision parameter $\xi$ (in the illustrative example it is the mass per unit length of a floor component) while the cost of full damage $C_{\mathrm{D}}$ is independent of $\xi$.

If $C_{0}(\xi)$ is proportional to the decision parameter $\xi$, and the load effect $S$ is proportional to a power $\xi^{-k}(k \geq 1)$, then the optimum ratio $C_{\mathrm{D}} / C_{0}(\xi)$ may be expressed $[4,5]$ as

$\frac{C_{\mathrm{D}}}{C_{0}(\xi)}=\left(k \frac{\partial \pi(\xi)}{\partial \mu_{S}(\xi)} \mu_{S}(\xi)+(k+1) \frac{\partial \pi(\xi)}{\partial \sigma_{S}(\xi)} \sigma_{S}(\xi)\right)^{-1}$,

where the quantities $C_{0}(\xi), \mu_{S}(\xi), \sigma_{S}(\xi)$ are dependent on the decision parameter $\xi$. Partial derivatives of the fuzzy probability of failure $\pi$ in equation (4) are to be determined using equation (2) and numerical methods of integration and derivation.

\section{Vibration of a floor member}

Vibration of a load bearing horizontal member supporting the floor structure of a building may be analysed using the equation of motion [8] for a beam:

$$
\xi \frac{\partial^{2} v(z, t)}{\partial t^{2}}+E J \frac{\partial^{4} v(z, t)}{\partial z^{4}}=F(z, t),
$$

where $v(z, t)$ denotes the vertical deflection and $F(z, t)$ denotes a load function of a generic point $z$ and time $t, \xi$ denotes the mass of the beam per unit length and $E J$ the stiffness of the beam. In the case of vibration criteria for building structures [7] ensuring human comfort, the relevant variable used to verify the serviceability conditions of a beam is the acceleration $a(z, t)$ which follows from equation (5) as

$a(z, t)=\frac{\partial^{2} v(z, t)}{\partial t^{2}}=\left(F(z, t)-E J \frac{\partial^{4} v(z, t)}{\partial z^{4}}\right) \xi^{-1}$.

If the decision parameter is the mass per unit length $\xi$, then the load effect $S$, being the root mean square value of acceleration, can be expressed in terms of $\xi$ as

$$
S=K(z) \xi^{-1},
$$

where $K(z)$ is a function expressing the shape of the deflection curve. Thus, in this case of vibration of a floor member, the load effect $S$ is proportional to $\xi^{-1}$ and the parameter $k$, entering equation (4), is equal to 1 . 


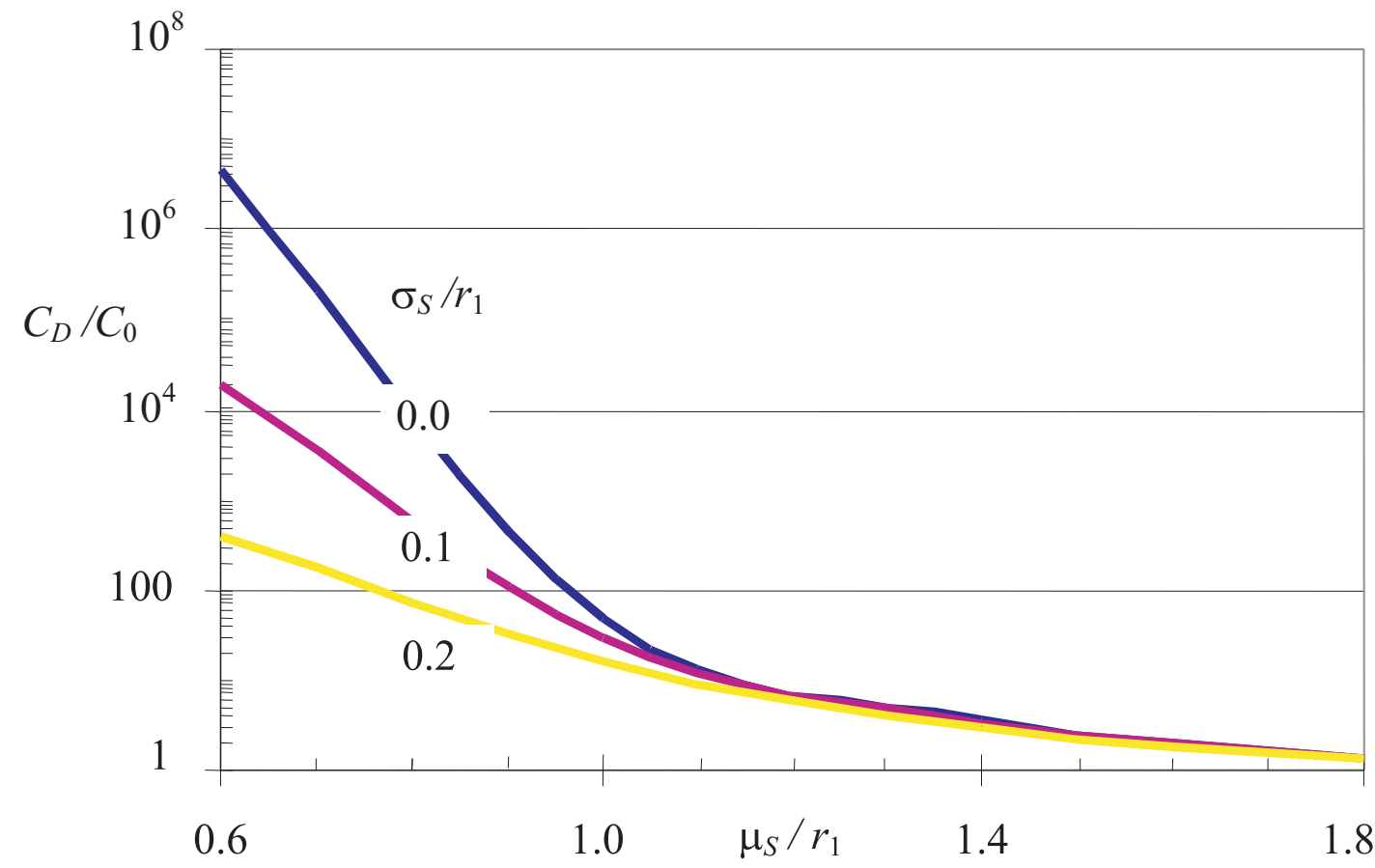

Fig. 2: Optimum cost ratio $C_{\mathrm{D}} / C_{0}$ for $k=1, \sigma_{R}=0.1 r_{1}$ and $r_{2} / r_{1}=3$

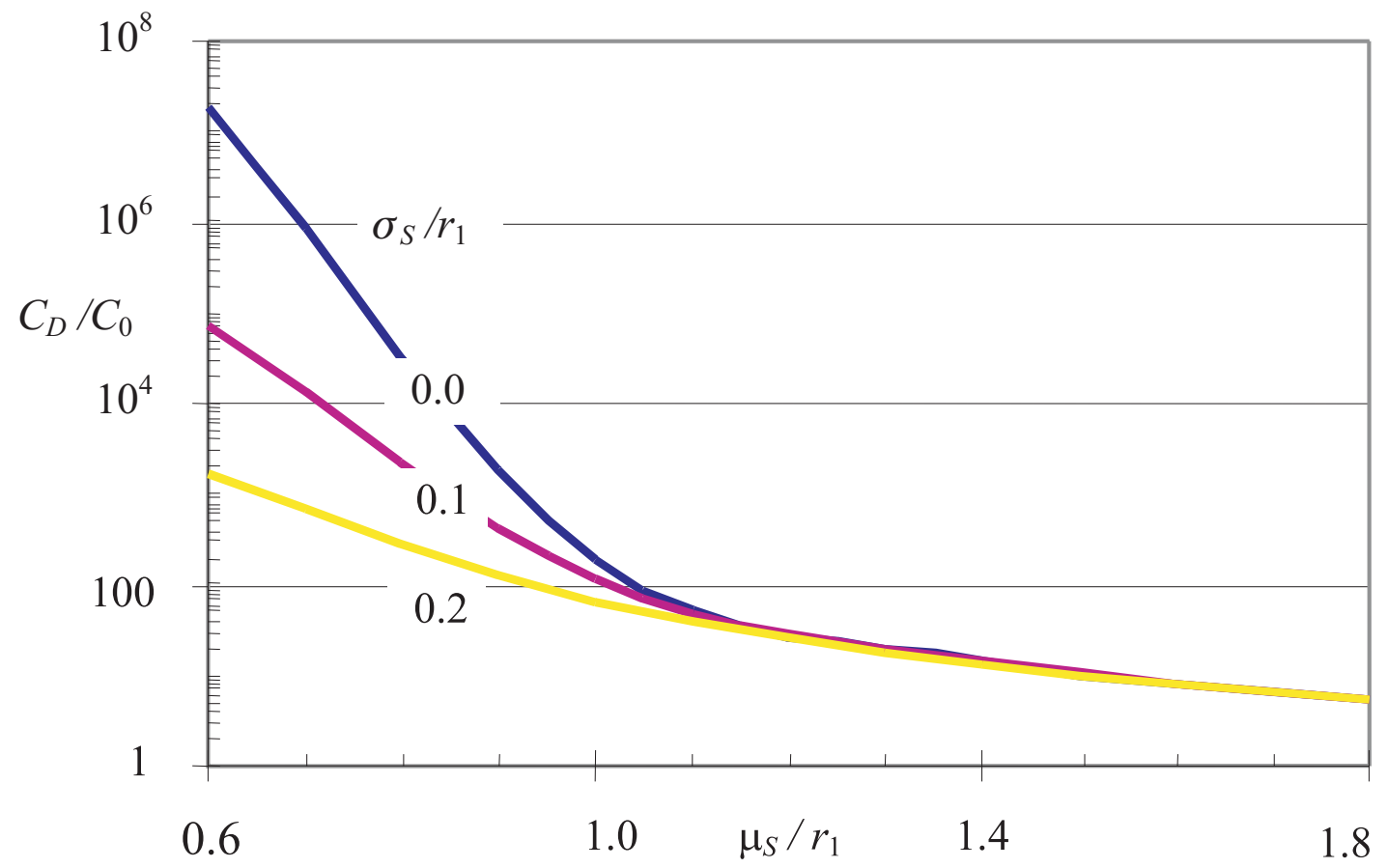

Fig. 3: Optimum cost ratio $C_{\mathrm{D}} / C_{0}$ for $k=1, \sigma_{R}=0.1 r_{1}$ and $r_{2} / r_{1}=5$

The optimum cost ratio $C_{\mathrm{D}} / C_{0}$ obtained from equation (4) for $k=1, \sigma_{R}=0.1 r_{1}$, and for $r_{2} / r_{1}=3$ is shown for selected values of $\mu_{S}$ and $\sigma_{S}$ in Fig. 2. Similar results are shown in Fig. 3 for $r_{2} / r_{1}=5$.

Assuming $\sigma_{R}=0.1 r_{1}$ and $r_{2} / r_{1}=5$ it follows from Fig. 3 that the optimum values of $C_{\mathrm{D}} / C_{0}$ are slightly higher than those corresponding to $\sigma_{R}=0.1 r_{1}$ and for $r_{2} / r_{1}=3$, which are indicated above in Figure 2. In both cases (for $r_{2} / r_{1}=3$ and $\left.r_{2} / r_{1}=5\right)$ the optimum cost ratio $C_{\mathrm{D}} / C_{0}$ for $\mu_{S}>r_{1}$ is very low (less than 100) and almost independent of the standard deviation $\sigma_{S}$.
It is interesting to note that the optimum probability ratio $\pi C_{\mathrm{D}} / C_{0}$ is almost independent of the characteristic of the load effect $S$ described by the mean $\mu_{S}$ and the standard deviation $\sigma_{S}$. Fig. 4 shows the variation of $\pi C_{\mathrm{D}} / C_{0}$ with $\mu_{S} / r_{1}$ for selected $\sigma_{S} / r_{1}$ assuming the same input data as in the illustrative example above: $k=1, \sigma_{R}=0.1 r_{1}$ and $r_{2} / r_{1}=3$. It should be noted that the resulting values for $k=1, \sigma_{R}=0.1 r_{1}$ and $r_{2} / r_{1}=5$ are almost exactly the same.

Considering $\mu_{S} / r_{1} \approx 0.2$ and $\sigma_{S} / r_{1} \approx 1$ it follows from Fig. 4 that the optimum fuzzy probability of performance failure may be assessed using an approximate relation 


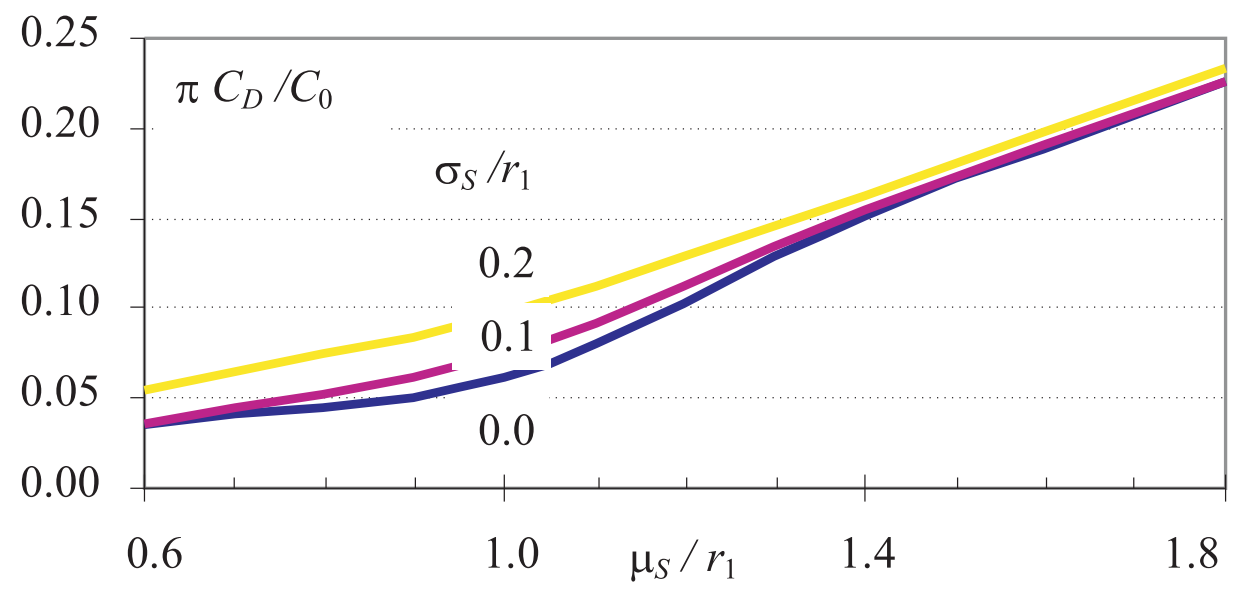

Fig. 4: Optimum probability ratio $\pi C_{\mathrm{D}} / C_{0}$ for $k=1, \sigma_{R}=0.1 r_{1}$ and $r_{2} / r_{1}=3$

$$
\pi \approx 0.1 \frac{C_{0}}{C_{\mathrm{D}}} .
$$

Fig. 4 may be used to adjust equation (8) for relevant values $\mu_{\mathrm{S}} / r_{1}$ and $\sigma_{S} / r_{1}$. Thus, equation (8) may be used for a first estimate of the optimum fuzzy probability of performance failure $\pi$ assuming that the action effect is described by $\mu_{S} / r_{1} \approx 0.2$ and $\sigma_{S} / r_{1} \approx 1$ and the performance constraint by $k=1, \sigma_{R}=0.1 r_{1}$ and by $r_{2} / r_{1}$ approximately from 3 to 5 . Then, having the costs $C_{0}$ and $C_{\mathrm{D}}$, the optimum fuzzy probability of performance failure $\pi$ may be assessed using equation (8). For example, if the expected cost ratio $C_{\mathrm{D}} / C_{0}=100$ (the damage cost $C_{\mathrm{D}}$ is hundred times greater than $C_{0}$, then the optimum $\pi$ is 0.001 .

It should be noted that the acceleration constraints required for various buildings (loading areas and activities) may significantly vary (in particular the lower and upper limits $r_{1}$ and $r_{2}$ may be related in various ways) [8]. Furthermore, requirements concerning other aspects of structural performance may be characterised by input data dissimilar to those considered in Fig. $4[4,5]$. Then an additional analysis considering appropriate input data $k, \sigma_{R}$ and $r_{2} / r_{1}$ is needed.

\section{Discussion}

Fuzzy optimisation of structural performance is applicable to many types of serviceability and other functional requirements, particularly of those affected by significant vagueness (for example deflection and acceleration). An example of an acceleration constraint for continuous vibration of a structure in an office building illustrates the general concepts well. It appears that the lower and upper bounds for acceleration constraints, denoted here $r_{1}$ and $r_{2}$ respectively, may vary within a broad range. Similar conditions may be observed in the case of other serviceability indicators as the deflection or crack width.

Assuming $r_{2} / r_{1}=3$ and $\sigma_{R}=0.1 r_{1}$ it follows from Fig. 2 for the cost ratio $C_{\mathrm{D}} / C_{0}$ equal to about 100 , that the building should be designed in such a way that the characteristics of action effects should correspond to the horizontal line at the level $C_{\mathrm{D}} / C_{0}=100$; for example if $\sigma_{S}=0.1 r_{1}$, then the optimum mean is $\mu_{S}=0.9 r_{1}$ (which is equal to $0.018 \mathrm{~ms}^{-2}$ if $r_{1}=0.02 \mathrm{~ms}^{-2}$ and $r_{2} / r_{1}=3$ ). For $r_{2} / r_{1}=5$ it follows from Fig. 3 that for $\sigma_{R}=\sigma_{S}=0.1 r_{1}$ the optimum mean is $\mu_{S}=r_{1}$ (which is equal to $0.02 \mathrm{~ms}^{-2}$ ). Thus, with the increasing upper limit $r_{2}$ the optimum mean $\mu \mathrm{S}$ also increases (from 0.018 to 0.02 $\mathrm{ms}^{-2}$ ). Note that for the standard deviation $\sigma_{S}=0.2 r_{1}$ the optimum mean $\mu_{S}$ is in both cases considerably lower (from 0.8 $r_{1}$ to $\left.0.95 r_{1}\right)$.

If the cost ratio $C_{\mathrm{D}} / C_{0}$ is $10^{4}$, then for $r 2 / r_{1}=3$ (Fig. 2) and, as above for $\sigma_{R}=\sigma_{S}=0.1 r_{1}$, the optimum mean $\mu_{S}$ of the load effect is about $0.63 r_{1}\left(0.0136 \mathrm{~ms}^{-2}\right)$, for $r_{2} / r_{1}=5$ (Fig. 3) the optimum mean $\mu_{S}$ of the load effect is about $0.72 r_{1}$ $\left(0.0144 \mathrm{~m} \mathrm{~s}^{-2}\right)$. Again, with the increasing upper limit $r_{2}$ the optimum mean $\mu_{S}$ also increases (from 0.0136 to $0.0144 \mathrm{~ms}^{-2}$ ). For the standard deviation $\sigma_{S}=0.2 r_{1}$ the optimum mean $\mu_{S}$ is in both cases again considerably lower (less than $\left.0.4 r_{1}\right)$.

Generally, with the increasing cost ratio $C_{\mathrm{D}} / C_{0}$ and increasing the standard deviations $\sigma_{R}$ and $\sigma_{S}$ the optimum mean $\mu_{S}$ and the standard deviation $\sigma_{S}$ lead to decreases in of the action effect $S$. For higher values of these quantities the optimum values for the mean $\mu_{S}$ and the standard deviation $\sigma_{S}$ may be quite severe and may not be achievable without introducing adequate structural measures. In some cases it may be necessary to revise the overall design of the building. The acceleration constraints considered in various international documents $[1,6,7]$, which are generally greater than the lower limit $r_{1}$, correspond to the optimum cost ratio $C_{\mathrm{D}} / C_{0}$ in the range from 1 to 100 (see Fig. 2 and 3). In the case of office buildings such values of cost ratio seem to be rather low. Consequently, the values of acceleration constraints recommended in $[6,7,8]$ may be uneconomical. A similar observation was obtained in previous studies concerning deflections $[4,5]$.

Experience from the assessment of existing structures confirms that there is no distinct value that would uniquely distinguish acceptable and unacceptable structural conditions. The fuzzy probabilistic concept may well explain the disturbing variance in public perception and in expert assessments of the observed defects. In particular it was difficult to explain great differences in experts judgements. It appears that there is an optimum value of the performance indicator that would lead to the minimum total cost and may be considered as the most likely outcome of the expert assessment [10]. 
However, the presented concepts are based on merely hypothetical (although quite reasonable and plausible) assumptions concerning the theoretical models describing the randomness and vagueness of performance requirements. For example the acceleration constraints used in the case of vibration were derived from data available in the literature and standards that may not fully fit actual conditions. Obviously to make a more credible assessment of the optimum structural characteristics, appropriate experimental data enabling a realistic definition of the relevant theoretical models is needed. At present only limited data is available, and it will be difficult to obtain new data experimentally.

The most difficult problems seem to be connected with the definition of the membership function $v_{R}(x)$ and the specification of the relevant probability distributions, for which conceivable theoretical models are used only. In particular both the limits $r_{1}$ and $r_{2}$ and the type of function $v_{R}(x)$ (which may be a non-linear function of the indicator $x$ ) should be derived from appropriate experimental data. Nevertheless, general concepts and the developed methodical principles supplemented by auxiliary computer programs seem to provide effective tools for comparative studies and further investigation of the structural performance.

\section{Conclusions}

(1) Performance requirements on structural behaviour are generally affected by two types of uncertainty: randomness and vagueness due to indistinct or imprecise definitions and perceptions; the theory of probability and the theory of fuzzy sets may be used to analyse them.

(2) The newly developed fuzzy probabilistic concepts including the damage function and the fuzzy probability of performance failure provide effective measures enabling rational analysis and the optimisation of structural performance.

(3) The proposed fuzzy probabilistic concepts are confirmed by available experience from the assessment of new as well as existing structures. Fuzzy probabilistic concepts may well explain the disturbing variance in public perception and in expert assessments of existing structures.

(4) Optimisation analysis indicates that commonly used performance criteria including the acceleration constraints for continuous vibration of a structure in office buildings may be uneconomical. Similar observations were obtained by previous studies concerning deflections.

(5) Appropriate experimental data enabling the specification of more realistic theoretical models is needed for further development and practical applications of fuzzy probabilistic concepts, including the optimisation of structural performance.

\section{Acknowledgment}

This paper presents a part of the findings of the research project CEZ: J04/98:210000029 "Reliability and risk engineering of technological systems ", supported by the Ministry of Youth and Education of the Czech Republic.

\section{References}

[1] Holický, M., Östlund, L.: "Probabilistic Design Concept.” Proc. International colloquium IABSE: Structural serviceability of buildings, Göteborg, 1983, p. 91-98.

[2] Brown, C. B., Yao, J. T. P.: "Fuzzy Sets and Structural Engineering." Journal of structural engineering, Vol. 109 (1983), No. 5, p. 1211-1225.

[3] Shiraishi, N., Furuta, H.: "Structural Design as Fuzzy Decision model.” Proc. ICASP 4, Pitagora Editrice, Bologna, 1983, p. 741-752.

[4] Holický, M.: "Fuzzy Optimisation of Structural Reliability." Proc. ICOSSAR'93, A.A.Balkema, Rotterdam, 1994, p. 1379-1382.

[5] Holický, M.: "Fuzzy Probabilistic Optimisation of Building Performance." Proc. CIB-ASTM-ISO-RILEM International symposium application of the performance concept in building, Tel Aviv, 2001, p. 4-75 to 84 . (see also Automation in construction 8, No. 4, 1999; p. 437-443).

[6] ISO 2631-2: Evaluation of Human Exposure to whole/ Body Vibration - Part 2: Continuous and Shock-Induced Vibration in Buildings (1 to 80) Hz, 1989.

[7] ISO 10137: Basis for Design of Structures - Serviceability of Buildings Against Vibration, 1991.

[8] Bachmann, H., Ammann, W.: Vibration in Structures Induced by Man and Machines. IABSE, Zurich, 1987.

[9] Holický, M.: "Performance Deficiency of a Department Store - Case Study." Proc. Safety, risk and reliability Trends in engineering; Zürich: IABSE, 2001, p. 321-326.

[10] Holický, M.: "Structural Failure and Assessment of a Department Store." Second International Conference on Forensic Engineering. ICE, London, November 2001, p. 55-63.

Prof. Ing. Milan Holický, Ph.D., DrSc.

phone: +420 224353842

fax: + 420224355232

e-mail: Holicky@klok.cvut.cz

Czech Technical University in Prague

Klokner Institute

Šolínova 7

16608 Praha 6, Czech Republic 